\title{
Correlative Atomic Force and Transmission Electron Microscopy toward Applications of Atomic Force Microscopy to Heterogeneous Systems
}

\author{
Yutaro Yamada $^{1}$,Takamitsu Haruyama ${ }^{2}$, Katsuya Shimabukuro ${ }^{3}$ and Hiroki Konno ${ }^{2}$ \\ 1. Graduate School of Natural Science \& Technology, Kanazawa University, Kanazawa, Japan. \\ 2. Bio-AFM Frontier Research Center, Kanazawa University, Kanazawa, Japan. \\ 3. Department of Chemical and Biological Engineering, National College of Technology, Ube campus, \\ Japan.
}

Atomic force microscopy (AFM) has been one of powerful techniques to study structures of dry or wet biological samples at nano meter spatial resolution. Furthermore, advent of high-speed AFM (HS-AFM) has boosted its temporal resolution, thereby dynamic nature of biological molecules has started being captured. Those include walking motion of myosinV on actin filaments, structural modulation of bacteriorhodopsin, sequential conformation change in three catalytic subunits in F-type ATP synthase and more [1-3]. However, AFM lacks an ability to discern particular biomolecule, thus its application in heterogeneous system is still challenge. To solve this problem, we have decided to integrate immunoelectron microscopy (immune EM), a powerful method to find localizations of a targeted biomolecule by an antibody in heterogeneous system, to AFM.

A key to success in correlative AFM/TEM is to accurately correlate two images captured separately under different physical conditions. For this reason, we create a reference marker on cover slips to help relocation of the target area in a sample and demonstrate the usefulness of this marker for biomolecule observation. To prepare cover slips with a patterned thin layer of gold, a locater grid (NEM) was placed on a cover slip ( $24 \times 40 \mathrm{~mm}$ ) and gold was deposited onto it by evaporation in a vacuum evaporator as described previously [4]. To prevent the gold layer from falling off, the patterned cover slips were baked at $160^{\circ} \mathrm{C}$ for overnight to stabilize the gold layer. After the coverslips cooled down to room temperature, the small areas containing the locater grid pattern was cut into $2 \times 2 \mathrm{~mm}$ pieces by a diamond pen, then adhered onto the top of a AFM stage with nail polish (Fig.1). We first used actin filaments as a testing material in correlative AFM/TEM observation. The actin filaments were fixed onto the prepared AFM stage and the topological image was obtained (Fig. 2a). Then the position of the scanning area in AFM observation against the pattern was recorded. After AFM observation the coverslip was gently taken apart from the AFM glass stage, and was process to make the replica for TEM observation sample. In TEM, the same scanning area of actin filaments in AFM observation was readily confirmed by using gold pattern (Fig. 2b). Immunue EM observation was performed after the treatment of gold colloid secondary antibody to actin filaments through primary antibody (Fig. 3). The arrowheads in Fig. 3b show specific binding of gold colloid to actin filaments. Although the number of binding gold colloid to actin filaments is not high, the proportion of specific binding of gold colloids to actin filaments was clearly higher than that of non-specific binding.

In summary, our results showed the usefulness of the cover slips containing reference marker to observe the same area in correlative AFM/TEM. We also demonstrated the possibility of identification of the biomolecule species in AFM by integration of immune EM. To identify particular molecular species in heterogeneous systems with correlative AFM/TEM is the next challenge. 


\section{References:}

[1] N Kodera et al, Nature 468 (2010), p. 72.

[2] M Shibata et al, Nature Nanotech 5 (2010), p. 208.

[3] T Uchihashi et al, Science 333 (2011), p. 1279.

[4] T Svitkina et al, Methods Enzymol 298 (1998), p. 570.

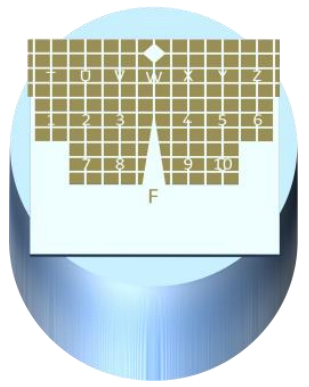

Figure 1. Schematic drawing of an AFM stage with gold-patterned coverslip for correlative AFM/TEM.

A gold-patterned coverslip $(2 \mu \mathrm{m} \times 2 \mu \mathrm{m})$ attached to the cylindrical glass stage on an AFM scanner.
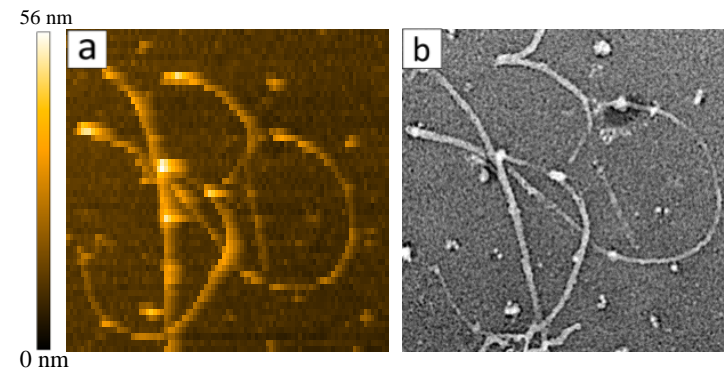

Figure 2. Observation of Actin filaments with HS-AFM and TEM.

(a) A HS-AFM image. Scan range, 3000 $\mathrm{nm} \times 3000 \mathrm{~nm}$. Scan rate, $4 \mathrm{~s} /$ frame._Z-scale, $56 \mathrm{~nm}$. (b) The TEM image of the same area in (a).

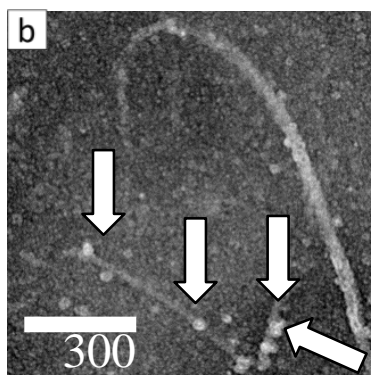

$0 \mathrm{~nm}$

Figure 3. HS-AFM and Immune-EM observation of actin filaments.

(a) HS-AFM image of actin filaments. Scan range, 3000 $\mathrm{nm} \times 3000 \mathrm{~nm}$. Scan rate, $6 \mathrm{~s} /$ frame. Z-scale, $103 \mathrm{~nm}$. (b) The TEM image of the same area in (a). Allows show gold colloidal particle that bound to actin filaments. 